\title{
Palladium Nanoparticles Supported on $\beta$-Cyclodextrin Functionalized Poly(amidoamine)s and Their Application in Suzuki-Miyaura Cross-Coupling Reactions
}

\author{
Wei Zhang, ${ }^{\circledR a}$ Zi-Jian Yao ${ }^{b}$ and Wei Deng* ${ }^{*, a, b}$ \\ ${ }^{a}$ School of Materials Science and Engineering, Shanghai University, Shang-Da Road, 99, \\ 200444 Shanghai, China \\ ${ }^{b}$ School of Chemical and Engineering, Shanghai Institute of Technology, Hai-Quan Road, 100, \\ 201418 Shanghai, China
}

\begin{abstract}
Herein, the synthesis, characterization and catalytic application of an organic-inorganic, palladium (Pd)-containing hybrid material, poly(amidoamine)-cyclodextrin (Pd@PAAs-CD), is reported as an efficient catalyst for Suzuki-Miyaura coupling reactions. The structure of Pd@PAAs-CD was confirmed by Fourier transform infrared spectroscopy (FTIR), transmission electron microscopy (TEM), scanning electron microscopy (SEM), inductively-coupled plasma atomic emission spectroscopy (ICP-AES), and ${ }^{1} \mathrm{H}$ nuclear magnetic resonance (NMR) spectroscopy. Furthermore, an efficient protocol has been developed using Pd@PAAs-CD as the catalyst in a Suzuki-Miyaura cross-coupling reaction in an aqueous medium in high yields. By using cyclodextrin (CD) as the mediator grafted onto PAAs, the Pd nanoparticles (NPs) were dispersed and enhanced the catalytic reaction by promoting host-guest interactions with the $\mathrm{CD}$. In addition, the reusability of the Pd@PAAs-CD hybrid material is demonstrated for use in multiple sequential cross-coupling reactions.
\end{abstract}

Keywords: $\beta$-cyclodextrin, catalysis, palladium nanoparticles, Suzuki-Miyaura cross coupling, poly(amidoamine)

\section{Introduction}

Over the past several decades, palladium-catalyzed cross-coupling reactions have become one of the most extensive and universal synthetic tools for the construction of $\mathrm{C}-\mathrm{C}$ bonds in organic synthesis. ${ }^{1-7}$ In particular, palladium-catalyzed Suzuki-Miyaura reactions have several demonstrated advantages, including mild reaction conditions, readily-available organic boron reagents, and tolerance of a variety of functional groups. ${ }^{8,9}$ In particular, aryl-aryl Suzuki-Miyaura cross coupling reactions are increasingly employed in the total synthesis of complex natural products ${ }^{10,11}$ and certain drug compounds. ${ }^{12}$ Macrocyclic supramolecules, such as crown ethers, $\mathrm{CD}$ (cyclodextrin), and calixarenes possess unique and size-tunable cavities that exhibit interesting properties. $\mathrm{CD}$ is a water-soluble, nontoxic organic macrocycle that is comprised of D-(+)-glucopyranose units (including $\alpha-, \beta-$, and $\gamma-C D) .{ }^{13-15} \beta-C D$ is the most widely-used of these macrocyclic compounds since it has a suitable

*e-mail: wdeng@shu.edu.cn cavity size and economical price. ${ }^{16-21}$ In recent years, organic-inorganic hybrid materials have attracted an increasing amount of attention from scientists. $22-26$ Organic-inorganic hybrid materials based on CD and noble metal nanoparticles have been widely researched and have shown applications in areas such as catalysis, small-molecule and gas detection, self-assembly, gene delivery, and as photoinitiators. ${ }^{27-32}$

Putta et al..$^{33}$ reported the use of palladium nanoparticles (Pd NPs)- $\beta$-CD-graphene nanosheets (Pd@CD-GNS) as the catalyst for $\mathrm{C}-\mathrm{C}$ coupling reactions and showed that this material exhibited excellent catalytic activity and dispersibility in water. Kaboudin et al. ${ }^{34}$ studied the preparation and catalytic activity of a novel $\mathrm{Pd}^{\mathrm{II}}-\beta$ CD complex in Suzuki-Miyaura coupling reactions. In addition, Gruttadauria and co-workers ${ }^{35}$ developed synthetic nanoparticle hybrids composed of poly(amidoamine) dendrimers and palladium (SWCNT-PAMAM 3.0-Pd) for the heterogeneous catalysis of Suzuki-Miyaura reactions. Monflier and co-workers ${ }^{36}$ designed ruthenium-containing $\beta$-cyclodextrin polymer globules that were applied to catalyze the hydrogenation of biomass-derived furanic compounds. 
In this paper, a simple and environmentally-friendly synthetic route is reported for the formation of Pd NPs via the direct reduction of $\mathrm{Pd}(\mathrm{OAc})_{2}$ by poly(amido amine)s-CD (PAAs-CD) and $\mathrm{NaBH}_{4}$ under mild reaction conditions. Since $\beta-C D$ is a water-soluble macrocycle, the addition of this compound allowed the Pd NPs to be evenly dispersed within the organic-inorganic hybrid materials. The structures of the synthesized Pd@PAAs-CD hybrids were confirmed using FTIR (Fourier transform infrared) spectroscopy, TEM (transmission electron microscopy), SEM (scanning electron microscopy), ICP-AES (inductively coupled plasma atomic emission spectroscopy), and ${ }^{1} \mathrm{H}$ NMR (nuclear magnetic resonance) spectroscopy. The hybrid material Pd@PAAs-CD exhibited excellent catalytic activity for Suzuki-Miyaura cross coupling reactions involving a diverse array of aryl halides in $\mathrm{H}_{2} \mathrm{O}$. Furthermore, selectivity experiments and the reusability of Pd@PAAs-CD were also studied in this paper.

\section{Experimental}

\section{Materials}

$\beta$-CD, palladium diacetate, ethanediamine, phenylboronic acid, and aryl halides were purchased from Adamas (China) and used without further purification. All experiments were carried out under argon atmosphere using standard Schlenk techniques. Solvents were used without further purification, and deionized water was used throughout the experiments.

\section{Procedure for the preparation of PAAs-CD}

PAAs based on mecysteine were prepared by the reaction of $N, N$-methylene bis(acrylamide) (MBA) and L-cysteine methyl ester hydrochloride (L-cys), by a procedure based on previous work. ${ }^{37}$ The polymerization was carried out under argon atmosphere, and a typical procedure is as follows: L-cys $(0.25 \mathrm{~g}, 1.5 \mathrm{mmol})$ was added to a mixture $(6 \mathrm{~mL}$, the volume ratio of water and dimethyl sulfoxide (DMSO) was 1:5) containing MBA $(0.45 \mathrm{~g}, 3.0 \mathrm{mmol})$. The polymerization was performed at $60{ }^{\circ} \mathrm{C}$ with vigorous stirring for $36 \mathrm{~h}$, when an excess of ethanediamine-CD (EDC-CD) 1.2 eq. per MBA was added, and the reaction was continued for an additional $36 \mathrm{~h}$. After this time, the solution was concentrated via rotary evaporation, and the resulting residue was precipitated into a $100 \mathrm{~mL}$ mixture of cold acetone/ether (5:1, v:v) with stirring. The crude product was further purified by re-precipitation from distilled water and acetone. Then, the product was dialysed (molecular cut off: $3.5 \mathrm{kD}$ ) for
$48 \mathrm{~h}$ (water changed every $12 \mathrm{~h}$ ). PAAs-CD was obtained by lyophilization and obtained in $87 \%$ yield.

\section{EDC-CD}

${ }^{1} \mathrm{H}$ NMR $\left(500 \mathrm{MHz}, \mathrm{D}_{2} \mathrm{O}-d_{2}\right) \delta 2.74(\mathrm{~s}, 2 \mathrm{H}), 2.79(\mathrm{~s}$, $2 \mathrm{H}), 3.50-3.65(\mathrm{~d}, 14.3 \mathrm{H}), 3.90(\mathrm{~d}, 28.0 \mathrm{H}), 5.05(\mathrm{~s}, 7.2 \mathrm{H})$.

\section{PAAs-CD}

${ }^{1} \mathrm{H}$ NMR (500 MHz, DMSO- $\left.d_{6}\right) \delta 0.74(\mathrm{~s}, 4.9 \mathrm{H}), 1.14$ (s, $8.5 \mathrm{H}), 2.30-2.87(\mathrm{~m}, 13.0 \mathrm{H}), 4.43(\mathrm{~d}, 4 \mathrm{H}), 4.8(\mathrm{~s}, 5 \mathrm{H})$, $5.59(\mathrm{~s}, 2.4 \mathrm{H}), 6.20(\mathrm{~d}, 2.8 \mathrm{H}), 8.50-8.68(\mathrm{~d}, 3.7 \mathrm{H})$.

\section{Procedure for the preparation of Pd@PAAs-CD}

The synthesis of Pd@PAAs-CD is described as follows: $100 \mathrm{mg}$ of PAAs-CD was dispersed into $10 \mathrm{~mL}$ DMSO and stirred at $50{ }^{\circ} \mathrm{C}$, and then $60 \mathrm{mg}$ of $\mathrm{Pd}(\mathrm{OAc})_{2}$ was added. The mixture was vigorously stirred, and the $\mathrm{NaBH}_{4}$ solution was added to promote the formation of Pd NPs, with continuous stirring for $18 \mathrm{~h}$.Pd@PAAs-CD precipitated from the solution upon addition of a mixture of acetone/ether (5:1, v:v). Pd@PAAs-CD was obtained via centrifugation $(6500 \mathrm{rpm} \times 3)$ and was then washed three times with water and then lyophilized for $24 \mathrm{~h}$ ( $80 \%$ yield). ICP-AES analysis was performed and showed that the palladium content of Pd@PAAs-CD catalyst was 10.77 wt.\%.

\section{Suzuki-Miyaura cross-coupling reaction}

A mixture of aryl halide $(0.25 \mathrm{mmol})$, phenylboronic acid (0.3 mmol), $\mathrm{Na}_{2} \mathrm{CO}_{3}(0.25 \mathrm{mmol}), \mathrm{PPh}_{3}(0.025 \mathrm{mmol})$ and Pd@PAAs-CD (0.5 mg, 0.2 mol\% Pd per mol of aryl halide) were placed into a $10 \mathrm{~mL}$ Schlenck tube with $1 \mathrm{~mL} \mathrm{H}_{2} \mathrm{O}$. The reaction mixture was reacted at $80^{\circ} \mathrm{C}$ for $18 \mathrm{~h}$, and the reaction progress was monitored via gas chromatography mass spectrometry (GC-MS). The reaction mixture was allowed to cool to room temperature, and then $3 \mathrm{~mL}$ water was added, and the product was extracted with ethyl acetate $(3 \mathrm{~mL} \times 3)$. The organic layers were combined and dried over anhydrous $\mathrm{Na}_{2} \mathrm{SO}_{4}$ and solvents were evaporated via rotary evaporator. The crude product was purified by column chromatography (200-300 mesh silica) using a mixture of petroleum ether/ethyl acetate $(10: 1, \mathrm{v}: \mathrm{v})$ as eluent. The structures of the products were confirmed by ${ }^{1} \mathrm{H}$ NMR and ${ }^{13} \mathrm{C}$ NMR spectroscopies.

\section{Spectral data for coupling products}

\section{Compound 3a}

White solid; ${ }^{1} \mathrm{H}$ NMR $\left(500 \mathrm{MHz}, \mathrm{CDCl}_{3}\right) \delta 1.45(\mathrm{t}, 3 \mathrm{H}$, $J 6.9 \mathrm{~Hz}, \mathrm{OCH}_{2} \mathrm{CH}_{3}$ ), 4.01 (q, $2 \mathrm{H}, J 6.9 \mathrm{~Hz}, \mathrm{OCH}_{2} \mathrm{CH}_{3}$ ), 
7.42 (t, 2H, J 7.6 Hz, $\left.\mathrm{CH}_{2}\right), 7.53$ (d, 2H, J $8.5 \mathrm{~Hz}, \mathrm{CH}_{2}$ ), $7.56(\mathrm{~m}, 5 \mathrm{H}, \mathrm{Ph}-\mathrm{H}) ;{ }^{13} \mathrm{C}$ NMR $\left(500 \mathrm{MHz}, \mathrm{CDCl}_{3}\right) \delta 14.91$, $63.55,114.80,115.05,126.73,128.14,128.74,132.10$, $132.17,140.89,158.54$.

\section{Compound $\mathbf{3 b}$}

White solid; ${ }^{1} \mathrm{H}$ NMR (500 MHz, $\left.\mathrm{CDCl}_{3}\right) \delta 6.78(\mathrm{~d}, 1 \mathrm{H}$, $J 7.5 \mathrm{~Hz}, \mathrm{CH}), 6.83(\mathrm{t}, 1 \mathrm{H}, J 7.9 \mathrm{~Hz}, \mathrm{CH}), 7.13(\mathrm{~m}, 2 \mathrm{H}$, $\left.\mathrm{CH}_{2}\right), 7.35(\mathrm{~m}, 1 \mathrm{H}, \mathrm{CH}), 7.45\left(\mathrm{~m}, 4 \mathrm{H}, \mathrm{CH}_{2}\right)$.

\section{Compound 3c}

White solid; ' ${ }^{1} \mathrm{H}$ NMR $\left(500 \mathrm{MHz}, \mathrm{CDCl}_{3}\right) \delta 3.75$ (s, 3H, $\left.\mathrm{OCH}_{3}\right), 6.83(\mathrm{~m}, 1 \mathrm{H}, \mathrm{CH}), 7.15\left(\mathrm{~m}, 2 \mathrm{H}, \mathrm{CH}_{2}\right), 7.27(\mathrm{~m}, 2 \mathrm{H}$, $\left.\mathrm{CH}_{2}\right), 7.38\left(\mathrm{~m}, 2 \mathrm{H}, \mathrm{CH}_{2}\right), 7.55\left(\mathrm{~m}, 2 \mathrm{H}, \mathrm{CH}_{2}\right) ;{ }^{13} \mathrm{C} \mathrm{NMR}$ $\left(500 \mathrm{MHz}, \mathrm{CDCl}_{3}\right) \delta 55.31,112.69,112.92,119.70,127.21$, $127.42,128.74,129.75,141.12,142.81,159.96$.

\section{Compound 3d}

White solid; ${ }^{1} \mathrm{H}$ NMR $\left(500 \mathrm{MHz}, \mathrm{CDCl}_{3}\right) \delta 6.70(\mathrm{~d}, 2 \mathrm{H}$, $\left.J 8.2 \mathrm{~Hz}, \mathrm{CH}_{2}\right), 7.18(\mathrm{~m}, 1 \mathrm{H}, \mathrm{CH}), 7.34\left(\mathrm{~m}, 4 \mathrm{H}, \mathrm{CH}_{2}\right), 7.46$ (d, $4 \mathrm{H}, J 7.3 \mathrm{~Hz}, \mathrm{CH}_{2}$ ).

\section{Compound 3e}

White solid; ${ }^{1} \mathrm{H}$ NMR $\left(500 \mathrm{MHz}, \mathrm{CDCl}_{3}\right) \delta 7.36(\mathrm{~m}, 2 \mathrm{H}$, Ar), 7.47 (t, 4H, J 7.3 Hz, Ar), 7.70-7.66 (d, 4H, J 7.5 Hz, $\mathrm{Ar}), 7.66$ (s, 4H, Ar).

\section{Compound $\mathbf{3 f}$}

White solid; ${ }^{1} \mathrm{H}$ NMR $\left(500 \mathrm{MHz}, \mathrm{CDCl}_{3}\right) \delta 6.72(\mathrm{~d}$, $J 8.2 \mathrm{~Hz}, \mathrm{Ar}$ ), 7.19 (m, 1H, Ar), 7.35 (m, 4H, Ar), 7.47 (d, $4 \mathrm{H}, J 7.3 \mathrm{~Hz}, \mathrm{Ar})$.

\section{Compound $\mathbf{3 g}$}

White solid; ${ }^{1} \mathrm{H}$ NMR $\left(500 \mathrm{MHz}, \mathrm{CDCl}_{3}\right) \delta 3.87$ (s, $\left.2 \mathrm{H}, \mathrm{CH}_{2}\right), 6.90-6.71(\mathrm{~d}, 2 \mathrm{H}, J 7.8 \mathrm{~Hz}, \mathrm{Ar}), 7.16-7.12$ (d, $2 \mathrm{H}, J 7.2 \mathrm{~Hz}, \mathrm{Ar}), 7.16-7.17$ (m, 1H, Ar), 7.22-7.26 (t, $2 \mathrm{H}$, $J 7.5 \mathrm{~Hz}, \mathrm{Ar}), 7.30-7.35$ (d, 2H, $J 8.3 \mathrm{~Hz}, \mathrm{Ar}) ;{ }^{13} \mathrm{C} \mathrm{NMR}$ $\left(500 \mathrm{MHz}, \mathrm{CDCl}_{3}\right) \delta 41.34,119.98,126.35,128.61,128.90$, 130.70, 131.55, 140.14, 140.48 .

\section{Compound $3 \mathrm{~h}$}

White solid; ${ }^{1} \mathrm{H}$ NMR $\left(500 \mathrm{MHz}, \mathrm{CDCl}_{3}\right) \delta 7.45$ (q, $1 \mathrm{H}, J 7.2 \mathrm{~Hz}, \mathrm{Ar}), 7.50$ (t, 2H, J 7.4 Hz, Ar), $7.62(\mathrm{~d}, 2 \mathrm{H}$, $J 7.2 \mathrm{~Hz}, \mathrm{Ar}), \delta 7.74(\mathrm{q}, 2 \mathrm{H}, J 8.7 \mathrm{~Hz}, \mathrm{Ar}), 8.29(\mathrm{~d}, 2 \mathrm{H}$, $J 8.3 \mathrm{~Hz}, \mathrm{Ar}) ;{ }^{13} \mathrm{C}$ NMR $\left(500 \mathrm{MHz}, \mathrm{CDCl}_{3}\right) \delta 124.13$, 127.40, 127.80, 128.94, 129.18, 128.68, 138.79, 147.65 .

\section{Compound $\mathbf{3 i}$}

White solid; ${ }^{1} \mathrm{H}$ NMR $\left(500 \mathrm{MHz}, \mathrm{CDCl}_{3}\right) \delta 7.36(\mathrm{t}, 1 \mathrm{H}$, $J 7.2 \mathrm{~Hz}, \mathrm{Ar}$ ), 7.41 (m, 4H, Ar), 7.54 (m, 4H, Ar).

\section{Compound 3j}

White solid; ${ }^{1} \mathrm{H}$ NMR $\left(500 \mathrm{MHz}, \mathrm{CDCl}_{3}\right) \delta 7.44$ (q, $1 \mathrm{H} J 7.3 \mathrm{~Hz}, \mathrm{Ar}), 7.48$ (t, 2H J 7.4 Hz, Ar), $7.58(\mathrm{~d}, 2 \mathrm{H}$, $J 7.3 \mathrm{~Hz}, \mathrm{Ar}$ ), 7.69 (d, 4H J 8.4 Hz, Ar), 7.78 (d, 4H J 21.6, $8.4 \mathrm{~Hz}, \mathrm{Ar}) ;{ }^{13} \mathrm{C} \mathrm{NMR}\left(500 \mathrm{MHz}, \mathrm{CDCl}_{3}\right) \delta 110.92,118.96$, $127.25,127.75,128.65,129.13,132.62,133.68,138.54$, 139.19, 145.69 .

\section{Compound 3/}

White solid; ${ }^{1} \mathrm{H}$ NMR $\left(500 \mathrm{MHz}, \mathrm{CDCl}_{3}\right) \delta 3.85$ (s, 3H, $\left.\mathrm{OCH}_{3}\right), 6.99$ (d, $\left.2 \mathrm{H}, J 8.5 \mathrm{~Hz}, \mathrm{Ar}\right), 7.30$ (t, $1 \mathrm{H}, J 7.4 \mathrm{~Hz}$, $\mathrm{Ar}), 7.42$ (t, $2 \mathrm{H}, J 7.5 \mathrm{~Hz}, \mathrm{Ar}), 7.56$ (t, 4H, Ar); ${ }^{13} \mathrm{C} \mathrm{NMR}$ $\left(500 \mathrm{MHz}, \mathrm{CDCl}_{3}\right) \delta 55.36,114.22,126.67,126.76,128.17$, $128.73,133.80,140.85,159.16$.

\section{Compound 3m}

White solid; ${ }^{1} \mathrm{H}$ NMR $\left(500 \mathrm{MHz}, \mathrm{CDCl}_{3}\right) \delta 7.14(\mathrm{t}, 2 \mathrm{H}$, $J 8.4 \mathrm{~Hz}, \mathrm{Ar}), 7.35(\mathrm{t}, 1 \mathrm{H}, J 7.2 \mathrm{~Hz}, \mathrm{Ar}), 7.44(\mathrm{t}, 2 \mathrm{H}, J 7.4 \mathrm{~Hz}$, $\mathrm{Ar}), 7.55(\mathrm{~d}, 4 \mathrm{H}, J 7.0 \mathrm{~Hz}, \mathrm{Ar}) ;{ }^{13} \mathrm{C} \mathrm{NMR}\left(500 \mathrm{MHz}, \mathrm{CDCl}_{3}\right)$ $\delta 115.55,115.72,127.05,127.19,127.28,128.68,128.74$, 128.84, 137.35, 140.28, 161.51, 163.47 .

\section{Compound $4 \mathbf{a}$}

White solid; ${ }^{1} \mathrm{H}$ NMR $\left(500 \mathrm{MHz}, \mathrm{CDCl}_{3}\right) \delta 1.44(\mathrm{t}, 3 \mathrm{H}$, $\left.J 6.9 \mathrm{~Hz}, \mathrm{OCH}_{2} \underline{\mathrm{CH}}_{3}\right), 4.06$ (q, $2 \mathrm{H}, J 6.9 \mathrm{~Hz}, \mathrm{OCH}_{2} \mathrm{CH}_{3}$ ), $6.97(\mathrm{~d}, 2 \mathrm{H}, J 8.2 \mathrm{~Hz}, \mathrm{Ar}), 7.38$ (d, 2H, J $8.2 \mathrm{~Hz}, \mathrm{Ar}), 7.46$ (d, 4H, J 7.8 Hz, Ar).

\section{Compound 4c}

White solid; ${ }^{1} \mathrm{H}$ NMR $\left(500 \mathrm{MHz}, \mathrm{CDCl}_{3}\right) \delta 3.86$ (s, 3H, $\left.J 6.9 \mathrm{~Hz}, \mathrm{OCH}_{3}\right), 6.92(\mathrm{~m}, 1 \mathrm{H}, \mathrm{Ar}), 7.08(\mathrm{~s}, 1 \mathrm{H}, \mathrm{Ar}), 7.15$ (d, 1H, J 7.6 Hz, Ar), 7.37 (t, 1H, J 7.9 Hz, Ar), 7.44 (d, $2 \mathrm{H}, J 8.4 \mathrm{~Hz}, \mathrm{Ar}), 7.60$ (d, 2H, J 8.5 Hz, $\mathrm{CH}_{2}$ ); ${ }^{13} \mathrm{C} \mathrm{NMR}$ $\left(500 \mathrm{MHz}, \mathrm{CDCl}_{3}\right) \delta 55.34,112.83,112.93,119.50,128.45$, $128.90,128.92,133.52,139.54,141.52,160.03$.

\section{Compound 4d}

White solid; ${ }^{1} \mathrm{H}$ NMR (500 MHz, $\left.\mathrm{CDCl}_{3}\right) \delta 3.66$ (br, $\left.2 \mathrm{H}, \mathrm{NH}_{2}\right), 6.68(\mathrm{~d}, J 8.2 \mathrm{~Hz}, \mathrm{Ar}), 7.29(\mathrm{t}, 4 \mathrm{H}, J 9.2 \mathrm{~Hz}, \mathrm{Ar})$, 7.37 (d, $2 \mathrm{H}, J 8.5 \mathrm{~Hz}, \mathrm{Ar}) ;{ }^{13} \mathrm{C}$ NMR $\left(500 \mathrm{MHz}, \mathrm{CDCl}_{3}\right.$ ) $\delta 115.43,116.74,127.60,127.89,128.78,130.26,132.04$, 132.17, 139.63, 146.13.

\section{Compound $\mathbf{4 f}$}

White solid; ${ }^{1} \mathrm{H}$ NMR (500 MHz, $\left.\mathrm{CDCl}_{3}\right) \delta 3.67$ (s, 2H, $\mathrm{NH}_{2}$ ), $6.68(\mathrm{~d}, 2 \mathrm{H}, J 8.2 \mathrm{~Hz}, \mathrm{Ar}), 7.29(\mathrm{t}, 4 \mathrm{H}, J 9.2 \mathrm{~Hz}, \mathrm{Ar})$, 7.37 (d, 2H, J 8.5 Hz, Ar).

\section{Compound $4 \mathbf{i}$}

White solid; ${ }^{1} \mathrm{H}$ NMR $\left(500 \mathrm{MHz}, \mathrm{CDCl}_{3}\right) \delta 7.41(\mathrm{~d}$, $4 \mathrm{H}, J 8.5 \mathrm{~Hz}, \mathrm{Ar}), 7.46$ (d, 4H, $J 8.4 \mathrm{~Hz}, \mathrm{Ar}) ;{ }^{13} \mathrm{C} \mathrm{NMR}$ 
$\left(500 \mathrm{MHz}, \mathrm{CDCl}_{3}\right) \delta 116.67,126.23,129.06,129.48$, 133.76, 128.14 .

\section{Compound $4 \mathrm{I}$}

White solid; ' ${ }^{1} \mathrm{H}$ NMR $\left(500 \mathrm{MHz}, \mathrm{CDCl}_{3}\right) \delta 3.83$ (s, 3H, $\left.\mathrm{OCH}_{3}\right)$ 6.80-6.95 (t, 2H, J 8.5 Hz, Ar), 7.31 (d, 2H, J $8.3 \mathrm{~Hz}$, Ar), 7.53 (t, 4H, J $8.2 \mathrm{~Hz}, \mathrm{Ar}$ ).

\section{Compound $4 p$}

White solid; ${ }^{1} \mathrm{H}$ NMR $\left(500 \mathrm{MHz}, \mathrm{CDCl}_{3}\right) \delta 7.41(\mathrm{~d}$, $4 \mathrm{H}, J 8.5 \mathrm{~Hz}, \mathrm{Ar}), 7.46$ (t, $4 \mathrm{H}, J 8.4 \mathrm{~Hz}, \mathrm{Ar}) ;{ }^{13} \mathrm{C} \mathrm{NMR}$ $\left(500 \mathrm{MHz}, \mathrm{CDCl}_{3}\right) \delta 116.67,128.23,129.06,129.48$, $133.76,138.44$.

\section{Compound 4s}

White solid; ${ }^{1} \mathrm{H}$ NMR $\left(500 \mathrm{MHz}, \mathrm{CDCl}_{3}\right) \delta 7.45(\mathrm{~d}$, $2 \mathrm{H}, J 8.2 \mathrm{~Hz}, \mathrm{Ar}), 7.54$ (d, 2H, J 8.3 Hz, Ar), 7.61 (t, $1 \mathrm{H}, J 7.7 \mathrm{~Hz}, \mathrm{Ar}), 7.81(\mathrm{~d}, 1 \mathrm{H}, J 7.6 \mathrm{~Hz}, \mathrm{Ar}), 7.86(\mathrm{~d}$, $1 \mathrm{H}, J 8.5 \mathrm{~Hz}, \mathrm{Ar}), 8.06$ (s, 1H, Ar), 10.8 (s, 1H, CHO); ${ }^{13} \mathrm{C} \mathrm{NMR}\left(500 \mathrm{MHz}, \mathrm{CDCl}_{3}\right) \delta 116.70,127.84,128.40$, 129.20, 129.65, 132.86, 134.27, 137.00, 138.14, 140.97, 192.21 .

\section{Compound $\mathbf{5 b}$}

White solid; ${ }^{1} \mathrm{H}$ NMR (500 MHz, $\left.\mathrm{CDCl}_{3}\right) \delta 1.44(\mathrm{t}, 3 \mathrm{H}$, $\left.J 6.9 \mathrm{~Hz}, \mathrm{OCH}_{2} \mathrm{CH}_{3}\right), 4.07$ (t, $2 \mathrm{H}, J 6.9 \mathrm{~Hz}, \mathrm{OCH}_{2} \mathrm{CH}_{3}$ ), $6.99(\mathrm{~d}, 2 \mathrm{H}, J 8.7 \mathrm{~Hz}, \mathrm{Ar}), 7.51$ (d, 2H, $J 8.7 \mathrm{~Hz}, \mathrm{Ar}), 7.64$ (d, $2 \mathrm{H}, J 8.2 \mathrm{~Hz}, \mathrm{Ar}), 7.67$ (d, 2H, J $8.2 \mathrm{~Hz}, \mathrm{Ar}$ ); ${ }^{13} \mathrm{C}$ NMR $\left(500 \mathrm{MHz}, \mathrm{CDCl}_{3}\right) \delta 14.81,63.63,110.05,115.08,119.13$, $127.08,128.33,132.57,132.90,145.28,159.62$.

\section{Compound 5c}

White solid; ' $\mathrm{H}$ NMR (500 MHz, $\left.\mathrm{CDCl}_{3}\right) \delta 3.87$ (3, $\left.3 \mathrm{H}, \mathrm{OCH}_{3}\right), 6.95-6.87$ (m, 1H, Ar), 7.10 (s, 1H, Ar), 7.17 (d, 1H, J 8.2 Hz, Ar), 7.17 (t, 1H, J 7.9 Hz, Ar), 7.68 (d, 2H, J $8.8 \mathrm{~Hz}, \mathrm{Ar}), 7.70$ (d, 2H, J 7.9 Hz, Ar); ${ }^{13} \mathrm{C} \mathrm{NMR}$ $\left(500 \mathrm{MHz}, \mathrm{CDCl}_{3}\right) \delta 55.40,113.11,113.91,119.68,127.81$, 130.19, 132.58, 140.66, 145.56, 160.15 .

\section{Compound 5d}

White solid; ${ }^{1} \mathrm{H}$ NMR $\left(500 \mathrm{MHz}, \mathrm{CDCl}_{3}\right) \delta 6.77(\mathrm{~d}$, $2 \mathrm{H}, J 7.8 \mathrm{~Hz}, \mathrm{Ar}), 7.43$ (d, 2H, J 7.8 Hz, Ar), 7.62 (d, 2H, $J 8.2 \mathrm{~Hz}, \mathrm{Ar}), 7.65$ (d, 2H, J $8.2 \mathrm{~Hz}, \mathrm{Ar})$.

\section{Compound $\mathbf{5 f}$}

White solid; ${ }^{1} \mathrm{H}$ NMR $\left(500 \mathrm{MHz}, \mathrm{CDCl}_{3}\right) \delta 6.76(\mathrm{~d}$, $2 \mathrm{H}, J 7.8 \mathrm{~Hz}, \mathrm{Ar}), 7.44$ (d, 2H, J 7.8 Hz, Ar), 7.64 (d, 2H, $J 8.2 \mathrm{~Hz}, \mathrm{Ar}), 7.64$ (d, 2H, J $8.2 \mathrm{~Hz}, \mathrm{Ar})$.

\section{Compound $\mathbf{5 g}$}

White solid; ${ }^{1} \mathrm{H}$ NMR $\left(500 \mathrm{MHz}, \mathrm{CDCl}_{3}\right) \delta 7.66(\mathrm{~m}$,
1H, Ar), 7.71 (d, 2H, J 8.2 Hz, Ar), 7.75 (d, 2H, J 8.6 Hz, Ar), 7.84 (d, 1H, J 7.9 Hz, Ar), 7.91 (d, 1H, J 7.6 Hz, Ar), 8.10 (s, 1H, Ar), 10.09 (s, 1H, CHO); ${ }^{13} \mathrm{C} \mathrm{NMR}(500 \mathrm{MHz}$, $\left.\mathrm{CDCl}_{3}\right) \delta 111.83,118.01,127.83,127.94,129.92,130.12$, 132.82, 132.90, 132.98, 137.15, 140.17, 144.15, 191.78.

\section{Compound $\mathbf{5 j}$}

White solid; ${ }^{1} \mathrm{H}$ NMR (500 MHz, $\left.\mathrm{CDCl}_{3}\right) \delta 7.69$ (d, 4H, $J 8.2 \mathrm{~Hz}, \mathrm{Ar}), 7.77$ (d, 4H, J 8.2 Hz, Ar); ${ }^{13} \mathrm{C}$ NMR (500 MHz, $\left.\mathrm{CDCl}_{3}\right) \delta 112.46,116.07,127.95,132.59,143.54$.

\section{Compound $\mathbf{5 I}$}

White solid; ${ }^{1} \mathrm{H}$ NMR $\left(500 \mathrm{MHz}, \mathrm{CDCl}_{3}\right) \delta 3.68(\mathrm{~s}, 3 \mathrm{H}$, $\left.\mathrm{OCH}_{3}\right), \delta 7.01(\mathrm{~d}, 2 \mathrm{H}, J 8.8 \mathrm{~Hz}, \mathrm{Ar}), 7.54(\mathrm{~d}, 2 \mathrm{H}, J 8.6 \mathrm{~Hz}$, Ar), 7.64 (d, 2H, J 8.5 Hz, Ar), 7.68 (d, 2H, J 8.5 Hz, Ar); ${ }^{13} \mathrm{C}$ NMR (500 MHz, $\mathrm{CDCl}_{3}$ ) $\delta 55.4,110.1,114.5,119.1$, $127.1,128.3,131.5,132.5,145.2,160.2$.

\section{Catalyst characterizations}

${ }^{1} \mathrm{HNMR}$ and ${ }^{13} \mathrm{C}$ NMR (500 MHz) spectra were recorded using a Bruker DMX-500 spectrometer (Switzerland). GC-MS data was obtained using a GC-MS QP2010-SE (Japan). The FTIR spectra were measured with a Nicolet FTIR spectrophotometer (USA) using $\mathrm{KBr}$ pellets in the spectral range $4000-400 \mathrm{~cm}^{-1}$. TEM experiments were performed on a JEOL 200CX (Japan), and SEM was measured using a JEOL LTD JSM-7500F (Japan). The Pd content of the samples was determined quantitatively by ICP-AES using a Thermo-Fisher Scientific iCAP 6300 instrument (England). Reagents were used without further purification, and deionized water was used throughout the experiments.

\section{Results and Discussion}

\section{Synthesis of Pd@PAAs-CD}

The experiment is divided into two parts: step 1 and step 2 (Scheme 1). The first step is a two-step (step 1-A and step 1-B), one-pot reaction. PAAs-CD was prepared via poly-condensation of MBA and L-cys in a 2:1 molar ratio (step 1-A) and then modified with excess ECD-CD (step 1-B). In step 1-A, MBA and L-cys reacted via a Michael polymerization to form branched PAAs, and the intermediate (PAAs) of the first step contained many olefin ends. The red circle is a zoom-in on its basic structure. In step 1-B, ECD-CD was added to aminate the PAAs. In the blue and green circles, the structure of the ECD-CD-loaded PAA is magnified. The CD binds to PAA through both Michael addition and carbonyl substitution. In step 2, 
PAAs-CD was dispersed in DMSO, and then $\mathrm{Pd}(\mathrm{OAc})_{2}$ was added. The mixture was continually stirred, and a solution of $\mathrm{NaBH}_{4}$ was added to promote the formation of Pd NPs. A precipitate containing Pd@PAAs-CD formed upon addition of a mixture of acetone and ether, which was then washed with water to give Pd@PAAs-CD. This precipitate was lyophilized to obtain Pd@PAAs-CD.

To verify the structure of the catalyst, a series of characterization experiments was performed. FTIR spectra of the Pd@PAAs-CD catalyst, EDC-CD, and PAAs are shown in Figure 1. In the PAAs sample, the absorption peaks at $3490,3322,3180$ and $2870 \mathrm{~cm}^{-1}$ are due to $\mathrm{N}-\mathrm{H}$ stretching vibrations from secondary amines, the $\mathrm{N}-\mathrm{H}$ stretching vibration of acid amides, and the $\mathrm{C}-\mathrm{H}$ stretching vibrations, respectively. The weak absorption peak located near $2450 \mathrm{~cm}^{-1}$ is attributed to the C-S bond. The FTIR data further confirmed the existence of PAAs and CD in the hybrid material Pd@PAAs-CD.

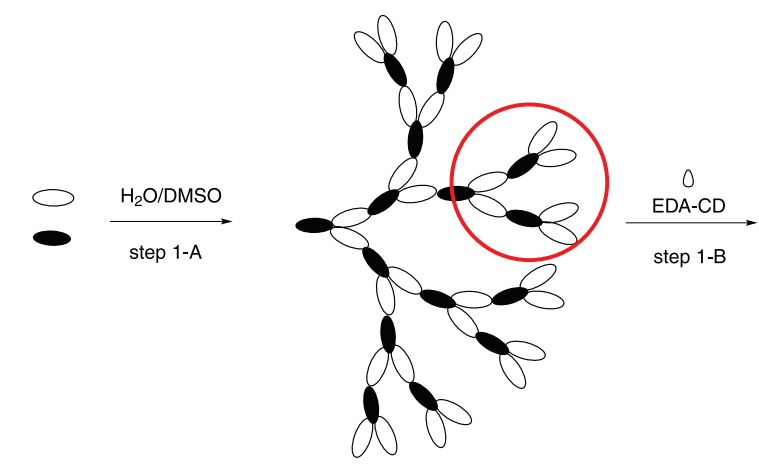

PAAs

$\boldsymbol{-}=\mathrm{HS}_{\mathrm{NH}_{2}}^{\mathrm{O}} \mathrm{O}^{-}$<smiles>C=CC(=O)NCNC(=O)C=C=C=O</smiles>

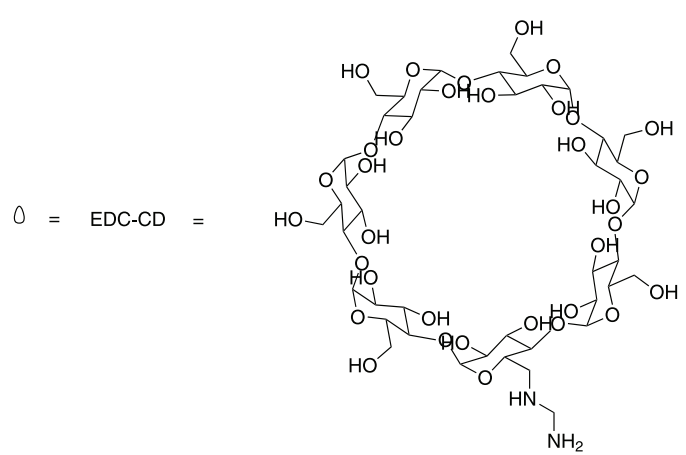

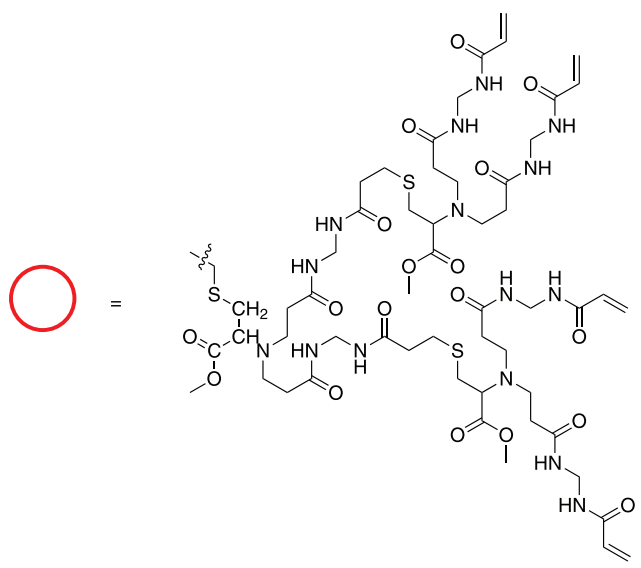

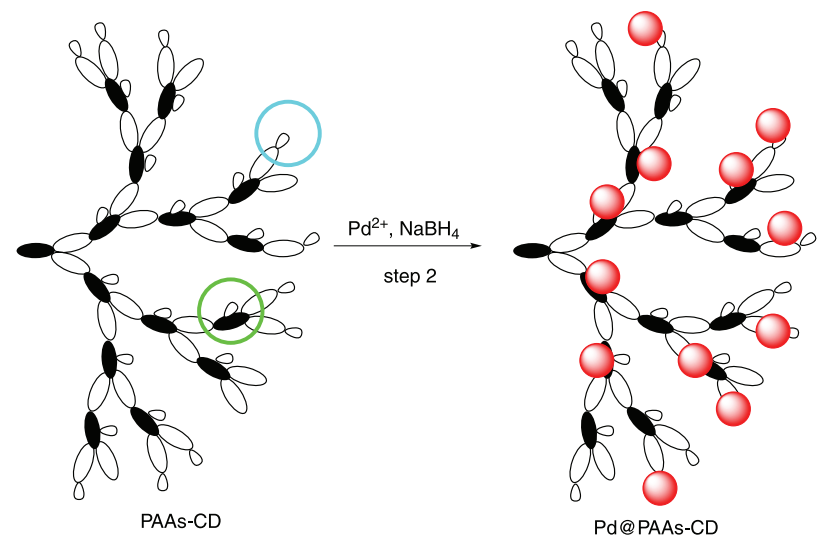

Pd@PAAs-CD

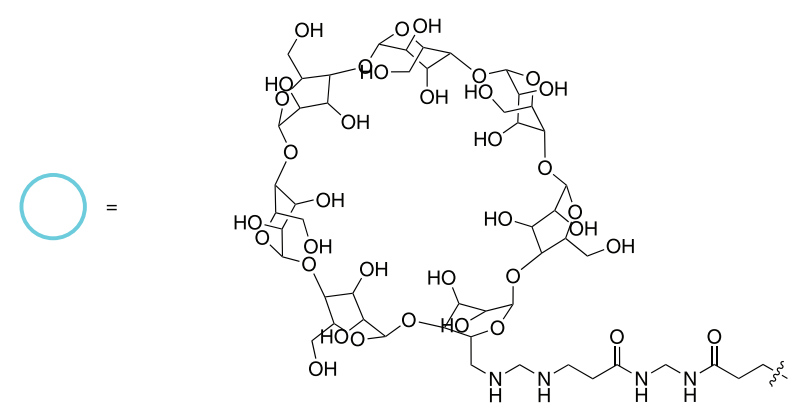

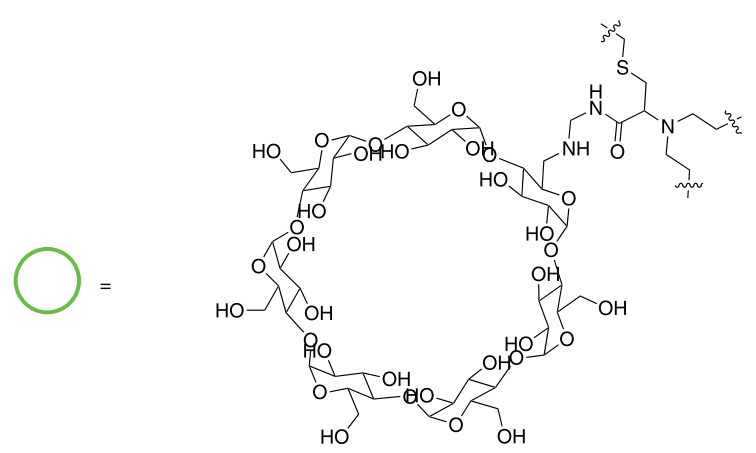

Scheme 1. Synthesis of Pd@PAAs-CD. 


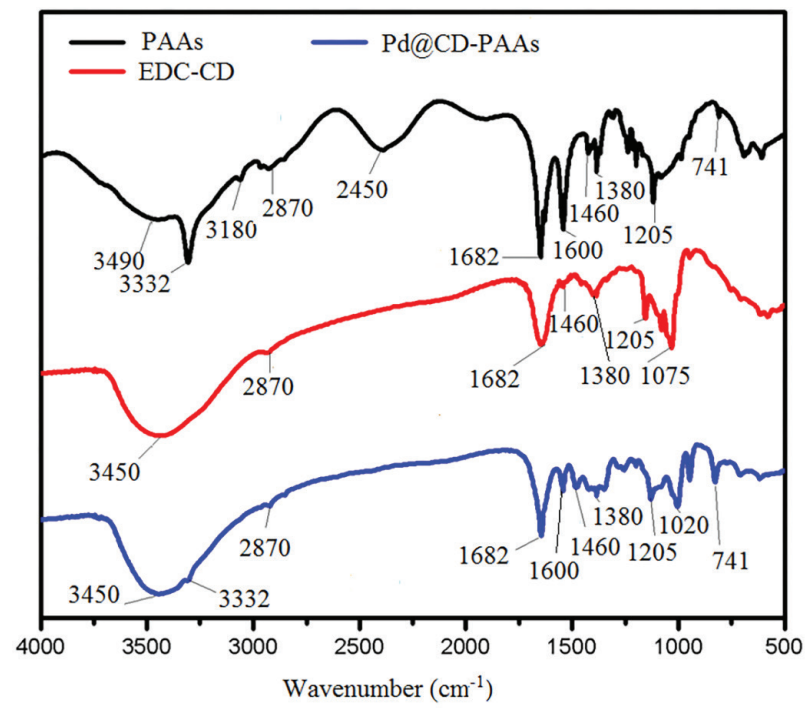

Figure 1. FTIR spectra of (a) PAAs (black) and (b) EDC-CD (red), (c) Pd@PAAs-CD (blue).

Figure 2 a shows the TEM image of CD-PAAs before Pd NPs loading, and Figure $2 b$ shows the TEM image of Pd@PAAs-CD that demonstrates the even dispersion of the Pd NPs onto the 10-15 nm PAAs-CD. The SEM image of Pd@PAAs-CD is shown in Figure 2c, which shows that the morphology of the Pd NPs has a globular radius of $10 \mathrm{~nm}$, indicating that the Pd NPs were uniformly dispersed onto the PAAs-CD.

Pd@PAAs-CD exhibited good dispersion and stability in water because of the introduction of $\mathrm{CD}$, which also improved the uniformity of the Pd NPs. The catalyst showed excellent catalytic activity in $\mathrm{C}-\mathrm{C}$ coupling reactions, likely due to the dispersibility of the Pd@PAAs-CD, which contained a variety of functional groups with lower catalyst loadings ( $\mathrm{Pd} 0.2 \mathrm{~mol} \%$ ) (Table 1, entries 1-10). After screening different bases, it was found that $\mathrm{Na}_{2} \mathrm{CO}_{3}$ gave the best yield (92\%), with $\mathrm{Cs}_{2} \mathrm{CO}_{3}$ giving a yield of $44 \%$, while $\mathrm{K}_{3} \mathrm{PO}_{4}$ resulted in a yield of $78 \%$ (Table 1 , entries $1,5,10)$. The reaction between 4-bromophenetole and phenylboronic acid afforded the product in $86 \%$ isolated yield with $\mathrm{Na}_{2} \mathrm{CO}_{3}$ (Table 1, entry 10). The temperature of $80{ }^{\circ} \mathrm{C}$ was determined to be the most suitable temperature for this reaction (Table 1, entries 11-14). When PAAs-CD was used as catalyst, no product was furnished (Table 1, entry 15), regardless of how much catalyst was added (Table 1, entries 16-17). A comparative experiment with the catalyst was also performed, but the yield was not ideal (Table 1, entry 18). These control experiments indicated the important role of $\mathrm{CD}$ in catalysis, which showed that using only $\mathrm{Pd}(\mathrm{OAc})_{2}$ under the same conditions was not ideal (Table 1, entry 19). After the catalytic cycle, the trivalent phosphine ligand was transformed into triphenyl phosphine oxide.

The selectivity of Pd@PAAs-CD was also investigated (Table 2), and the results show that the $\mathrm{Pd} / \mathrm{C}$ loaded with the same amount of palladium displays no catalytic activity (Table 2, entry 4). When the amount of $\mathrm{Pd} / \mathrm{C}$ was doubled, product yield was significantly improved, but by-products (mainly self-coupling products and raw materials) were also formed (Table 2, entry 5). When the amount of $\mathrm{Pd} / \mathrm{C}$ was tripled, the yield reached $87 \%$ (Table 2 , entry 6). When compared to Pd/C, the Pd@PAAs-CD exhibited a much higher catalytic efficiency, and in order to confirm that this increase in catalytic activity was due to addition of CD, a series of control experiments was performed. To the best of our knowledge, the binding of CD to amantadine has been widely reported. ${ }^{38-41}$ When amantadine was added to the solution of Pd@PAAs-CD, the catalytic effect was effectively inhibited (Table 2, entry 2), and no catalytic activity was observed even when the amount of catalyst was doubled (Table 2, entry 3). This was attributed to the fact that amantadine filled the cavity of $\mathrm{CD}$, which hindered the contact of guest molecules with Pd NPs and thus inhibited the activity of Pd@PAAs-CD. However, when amantadine was added to the solution of $\mathrm{Pd} / \mathrm{C}$, this compound still retained general activity (Table 2, entry 7). These

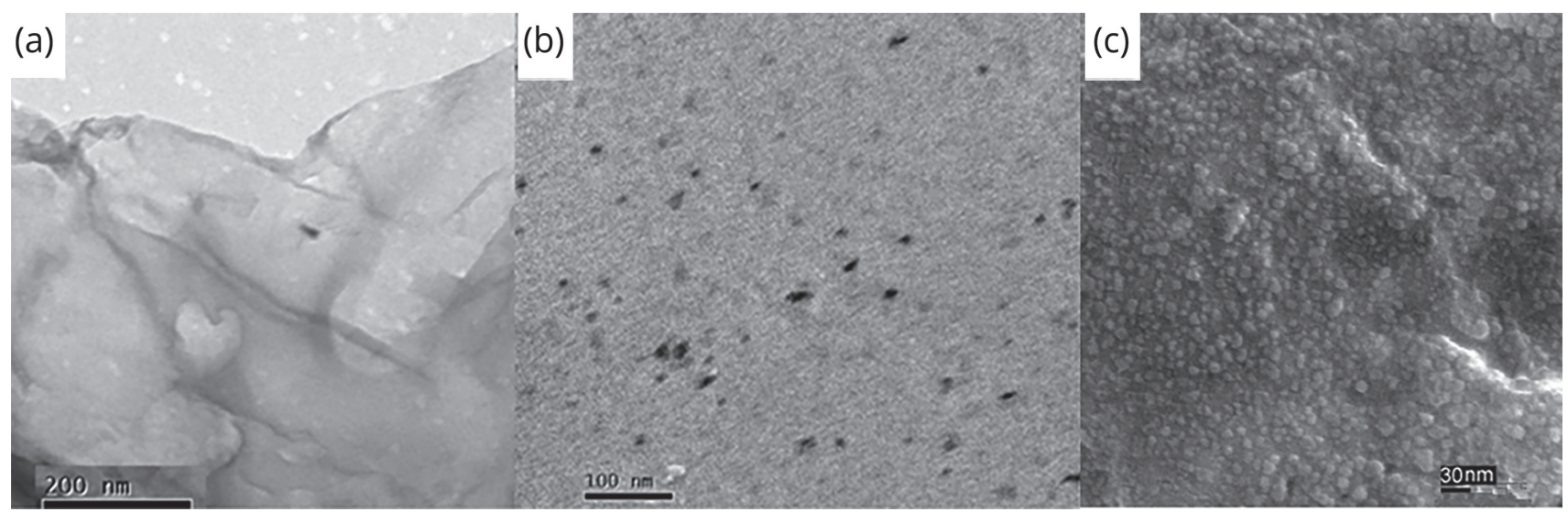

Figure 2. (a) TEM image of the PAAs-CD; (b) TEM image of the Pd@PAAs-CD; (c) SEM micrographs of Pd@PAAs-CD. 
Table 1. Screening of reaction conditions ${ }^{\mathrm{a}}$

\begin{tabular}{|c|c|c|c|c|c|}
\hline & $1 a$ & \multicolumn{4}{|c|}{$3 a$} \\
\hline entry & Catalyst & Base & Solvent & Temperature $/{ }^{\circ} \mathrm{C}$ & Yield $^{\mathrm{b}} / \%$ \\
\hline 1 & Pd@PAAs-CD & $\mathrm{Cs}_{2} \mathrm{CO}_{3}$ & $\mathrm{H}_{2} \mathrm{O}$ & 80 & 44 \\
\hline 2 & Pd@PAAs-CD & $\mathrm{K}_{2} \mathrm{CO}_{3}$ & $\mathrm{H}_{2} \mathrm{O}$ & 80 & 40 \\
\hline 3 & Pd@PAAs-CD & $\mathrm{Li}_{2} \mathrm{CO}_{3}$ & $\mathrm{H}_{2} \mathrm{O}$ & 80 & 75 \\
\hline 4 & Pd@PAAs-CD & $\mathrm{NaH}_{2} \mathrm{PO}_{4}$ & $\mathrm{H}_{2} \mathrm{O}$ & 80 & trace \\
\hline 5 & Pd@PAAs-CD & $\mathrm{K}_{3} \mathrm{PO}_{4}$ & $\mathrm{H}_{2} \mathrm{O}$ & 80 & 78 \\
\hline 6 & Pd@PAAs-CD & $\mathrm{NaHCO}_{3}$ & $\mathrm{H}_{2} \mathrm{O}$ & 80 & 80 \\
\hline 7 & Pd@PAAs-CD & $\mathrm{Na}_{3} \mathrm{PO}_{4}$ & $\mathrm{H}_{2} \mathrm{O}$ & 80 & 70 \\
\hline 8 & Pd@PAAs-CD & $\mathrm{K}_{2} \mathrm{HPO}_{4}$ & $\mathrm{H}_{2} \mathrm{O}$ & 80 & 50 \\
\hline 9 & Pd@PAAs-CD & triethylamine & $\mathrm{H}_{2} \mathrm{O}$ & 80 & 77 \\
\hline 10 & Pd@PAAs-CD & $\mathrm{Na}_{2} \mathrm{CO}_{3}$ & $\mathrm{H}_{2} \mathrm{O}$ & 80 & $92(86 \%)^{\mathrm{c}}$ \\
\hline 11 & Pd@PAAs-CD & $\mathrm{Na}_{2} \mathrm{CO}_{3}$ & $\mathrm{H}_{2} \mathrm{O}$ & r.t. & 5 \\
\hline 12 & Pd@PAAs-CD & $\mathrm{Na}_{2} \mathrm{CO}_{3}$ & $\mathrm{H}_{2} \mathrm{O}$ & 50 & 45 \\
\hline 13 & Pd@PAAs-CD & $\mathrm{Na}_{2} \mathrm{CO}_{3}$ & $\mathrm{H}_{2} \mathrm{O}$ & 100 & 84 \\
\hline 14 & Pd@PAAs-CD & $t$-BuOLi & $\mathrm{H}_{2} \mathrm{O}$ & 100 & 85 \\
\hline 15 & PAAs-CD & $\mathrm{Na}_{2} \mathrm{CO}_{3}$ & $\mathrm{H}_{2} \mathrm{O}$ & 80 & trace \\
\hline 16 & Pd@PAAs-CD & $\mathrm{Na}_{2} \mathrm{CO}_{3}$ & $\mathrm{H}_{2} \mathrm{O}$ & 80 & $92^{\mathrm{d}}$ \\
\hline 17 & Pd@PAAs-CD & $\mathrm{Na}_{2} \mathrm{CO}_{3}$ & $\mathrm{H}_{2} \mathrm{O}$ & 80 & $92^{\mathrm{e}}$ \\
\hline 18 & $\mathrm{Pd} @ \mathrm{PAAs}^{\mathrm{f}}$ & $\mathrm{Na}_{2} \mathrm{CO}_{3}$ & $\mathrm{H}_{2} \mathrm{O}$ & 80 & 65 \\
\hline 19 & $\mathrm{Pd}(\mathrm{OAc})_{2}{ }^{\mathrm{g}}$ & $\mathrm{Na}_{2} \mathrm{CO}_{3}$ & $\mathrm{H}_{2} \mathrm{O}$ & 80 & 6 \\
\hline
\end{tabular}

${ }^{a}$ Reaction conditions: aryl halide $(0.25 \mathrm{mmol})$, phenylboronic acid $(0.30 \mathrm{mmol}), \mathrm{PPh}_{3}(0.025 \mathrm{mmol})$, base $(0.25 \mathrm{mmol}), \mathrm{Pd} @ \mathrm{PAAs}-\mathrm{CD}(0.5 \mathrm{mg}$,

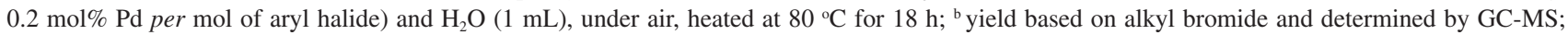
cisolated yield; ${ }^{\mathrm{Pd}} @ \mathrm{PAAs}-\mathrm{CD}(1 \mathrm{mg})$; ${ }^{\mathrm{e}} \mathrm{Pd} @ \mathrm{PAAs}-\mathrm{CD}(2 \mathrm{mg})$; ${ }^{\mathrm{f}} \mathrm{Pd} @ \mathrm{PAAs}\left(0.2 \mathrm{~mol} \% \mathrm{Pd}\right.$ per mol of aryl halide); ${ }^{\mathrm{g}} \mathrm{Pd}(\mathrm{OAc})_{2}(0.2 \mathrm{~mol} \% \mathrm{Pd}$ per mol of aryl halide). PAA: poly(amido amine); $\mathrm{CD}$ : cyclodextrin; r.t.: room temperature.

Table 2. Selective experiments of catalysts ${ }^{\mathrm{a}}$

\begin{tabular}{|c|c|c|c|}
\hline entry & Catalyst & Additive $^{b}$ & Yield $\mathrm{c} / \%$ \\
\hline 1 & Pd@PAAs-CD (Pd 0.2 mol\%) & - & 90 \\
\hline 2 & Pd@PAAs-CD (Pd 0.2 mol\%) & amantadine & trace \\
\hline 3 & Pd@PAAs-CD (Pd 0.6 mol\%) & amantadine & trace \\
\hline 4 & $\mathrm{Pd} / \mathrm{C}(\mathrm{Pd} 0.2 \mathrm{~mol} \%)$ & - & trace \\
\hline 5 & $\mathrm{Pd} / \mathrm{C}(\mathrm{Pd} 0.4 \mathrm{~mol} \%)$ & - & 55 \\
\hline 6 & $\mathrm{Pd} / \mathrm{C}(\mathrm{Pd} 0.6 \mathrm{~mol} \%)$ & - & 87 \\
\hline 7 & $\mathrm{Pd} / \mathrm{C}(\mathrm{Pd} 0.6 \mathrm{~mol} \%)$ & amantadine & 35 \\
\hline
\end{tabular}

Reaction conditions: aryl halide $(0.25 \mathrm{mmol})$, phenylboronic acid (0.30 mmol), $\mathrm{PPh}_{3}(0.025 \mathrm{mmol}), \mathrm{Na}_{2} \mathrm{CO}_{3}(0.25 \mathrm{mmol}), \mathrm{Pd} @ \mathrm{PAAs}-\mathrm{CD} /$ $(\mathrm{Pd} / \mathrm{C}) / \mathrm{PAAs}-\mathrm{CD}$ and $\mathrm{H}_{2} \mathrm{O}(1 \mathrm{~mL})$, under air, heated at $80{ }^{\circ} \mathrm{C}$ for $18 \mathrm{~h}$; bthe quality of amantadine is 10 times that of Pd@PAAs-CD/(Pd/C); cyield based on alkyl halide and determined by GC-MS. PAA: poly(amido amine); $\mathrm{CD}$ : cyclodextrin.

experiments show that using $\mathrm{CD}$ as the supramolecule could potentially improve the catalytic efficiency of Pd@PAAs-CD through host-guest interactions.
Reactions between phenylboronic acid and a variety of aryl halides were performed (Table 3), and the products were obtained in average to excellent yields. Even sterically-hindered aryl bromides substituted at the ortho- and meta- positions afforded 87 and $92 \%$ isolated yields when the reactions were run in pure water $(\mathbf{3 b}$ and 3c). The reaction with $p$-chloroaniline also achieved an average yield (3f). Notably, aryl halides with electrondonating amino, benzyl, bromomethyl, chloro, and methoxy substituents (3d, 3e, 3f, 3g, 3i, 3l, and 3m) gave the desired products in $80-90 \%$ yield. Electronwithdrawing groups, such as nitro, cyanide, and fluoro substituents $(\mathbf{3 h}, \mathbf{3} \mathbf{j}$, and $\mathbf{3 m})$ yielded products in 80 90\%. In addition, 2,4,6-trimethyliodobenzene with large amounts of steric hindrance (3k) gave products with a $44 \%$ yield. Unfortunately, aromatic alkanes and secondary aromatic bromides failed to give products in good yields using this catalyst system. 
Table 3. Suzuki-Miyaura cross coupling reactions between aryl halides and phenylboronic acid ${ }^{\mathrm{a}, \mathrm{b}}$

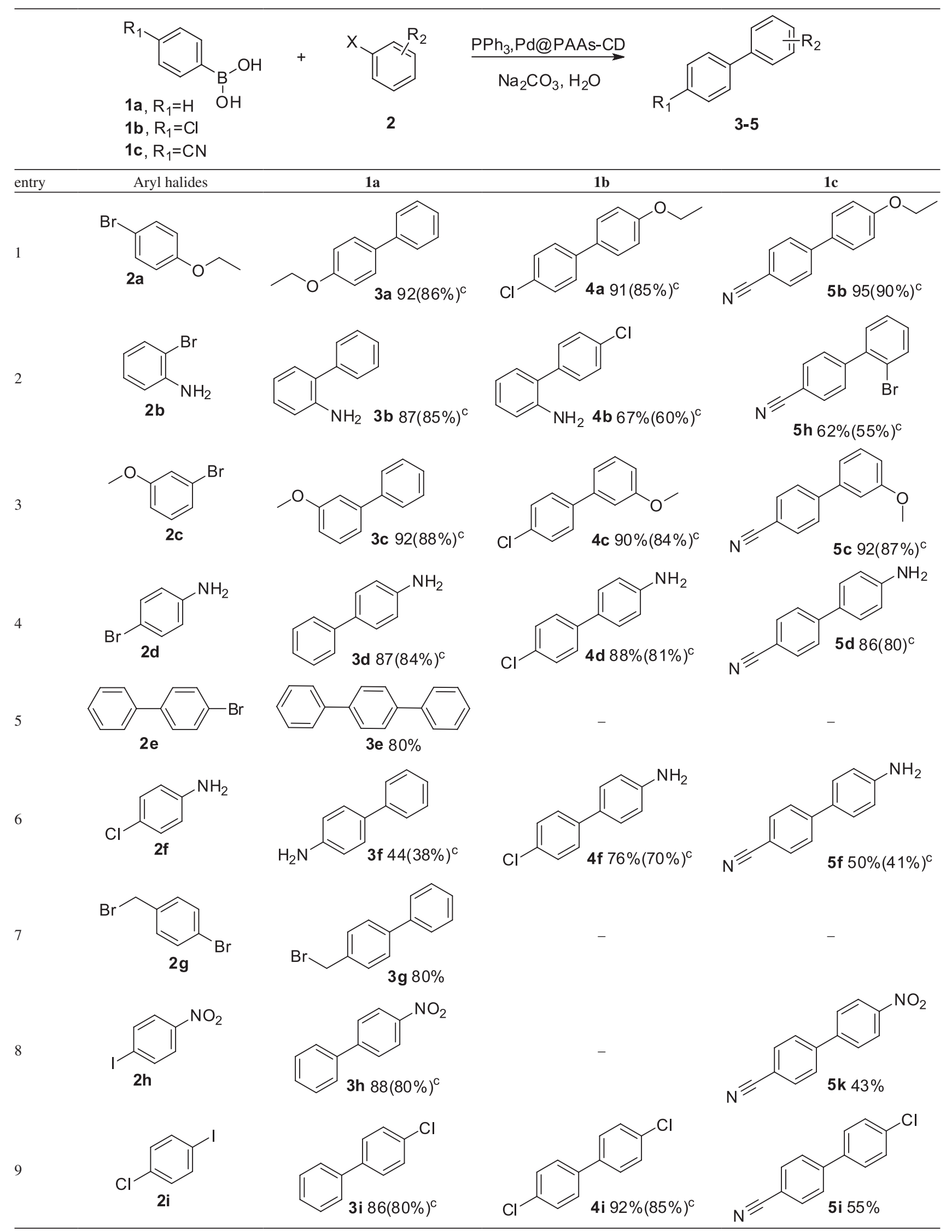


Table 3. Suzuki-Miyaura cross coupling reactions between aryl halides and phenylboronic acid ${ }^{\mathrm{a}, \mathrm{b}}$ (cont.)

entry

10<smiles>N#Cc1ccc(I)cc1</smiles>

2j

11<smiles>Cc1cc(C)c(I)c(I)c1</smiles>

12<smiles>COc1ccc(I)cc1</smiles>

13<smiles>Fc1ccc(I)cc1</smiles>

14<smiles>N#Cc1cccc(I)c1</smiles>

15<smiles>Clc1ccc(Br)cc1</smiles>

16<smiles>O=Cc1cccc(Br)c1</smiles>

2s

17<smiles>BrC1CCCCC1</smiles>

20

18<smiles>CCCCCCBr</smiles>

19<smiles>CC(Br)c1ccccc1</smiles>

20<smiles>CC(Cl)c1ccccc1</smiles>

\section{1b}<smiles>N#Cc1ccc(-c2ccccc2)cc1</smiles><smiles>COc1ccc(-c2ccc(Cl)cc2)cc1</smiles><smiles>COc1ccc(-c2ccc(C#N)cc2)cc1</smiles><smiles>Fc1ccc(-c2ccccc2)cc1</smiles><smiles>N#Cc1ccc(O)c(-c2ccccc2)c1</smiles><smiles>Clc1ccc(-c2ccc(Cl)cc2)cc1</smiles>
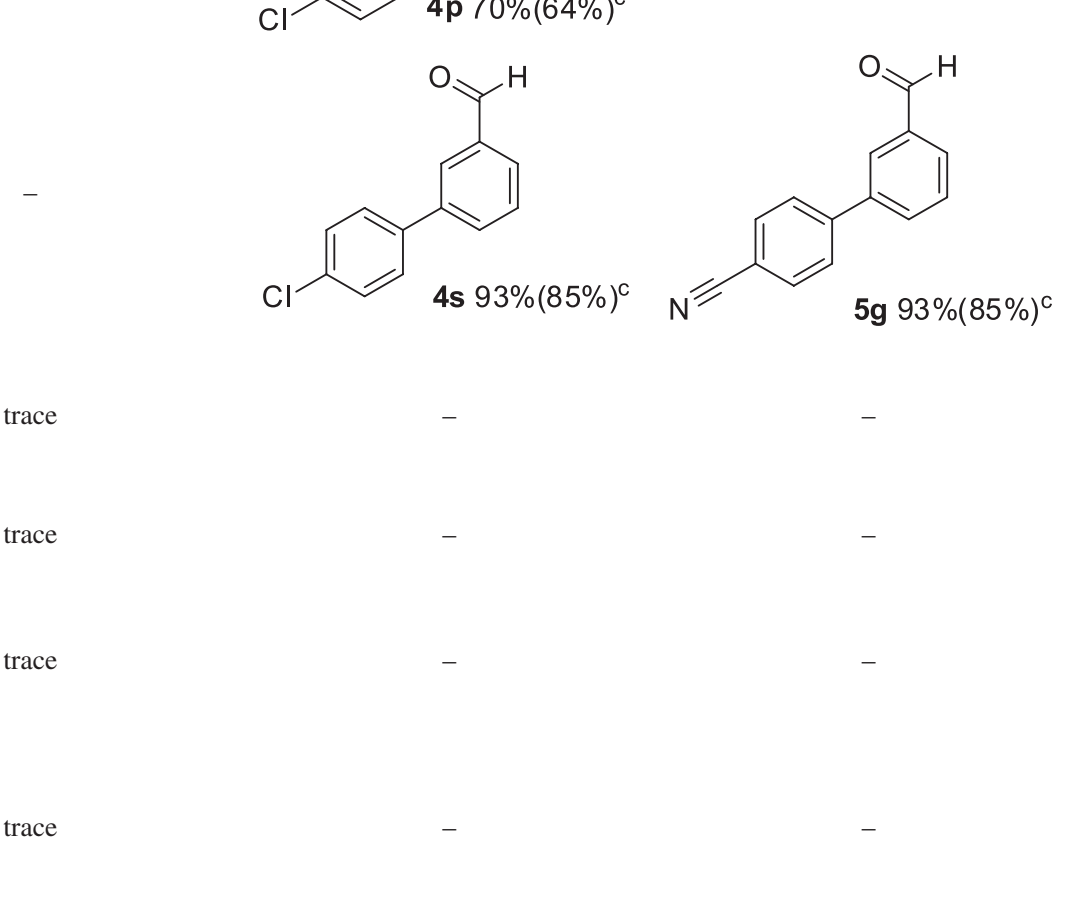

trace

trace

trace

trace<smiles>N#Cc1ccc(-c2ccc(C#N)cc2)cc1</smiles>

N

Reaction conditions: aryl halide (2) (0.25 mmol), phenylboronic acid (1a-1c) $(0.30 \mathrm{mmol}), \mathrm{PPh}_{3}(0.025 \mathrm{mmol}), \mathrm{Na}_{2} \mathrm{CO}_{3}(0.25 \mathrm{mmol}), \mathrm{Pd} @ \mathrm{PAAs}-\mathrm{CD}(0.5 \mathrm{mg}$,

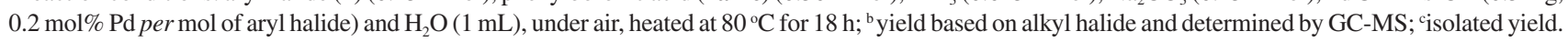


Table 4. Reusability of Pd@PAAs-CD

\begin{tabular}{lcccccc}
\hline No. of cycles & 1 & 2 & 3 & 4 & 5 & 6 \\
\hline Reaction conversion $/ \%$ & 90 & 87 & 85 & 82 & 81 & 79 \\
time / $\mathrm{h}$ & 18 & 18 & 18 & 18 & 18 & 18 \\
\hline
\end{tabular}

a Reaction conditions: $p$-bromoethylbenzene $(0.25 \mathrm{mmol})$, phenylboronic acid $(0.30 \mathrm{mmol}), \mathrm{PPh}_{3}(0.025 \mathrm{mmol}), \mathrm{Na}_{2} \mathrm{CO}_{3}(0.25 \mathrm{mmol}), \mathrm{Pd} @ \mathrm{PAAs}-\mathrm{CD}$ ( $0.5 \mathrm{mg}, 0.2 \mathrm{~mol} \% \mathrm{Pd}$ per mol of aryl halide) and $\mathrm{H}_{2} \mathrm{O}(1 \mathrm{~mL})$, under air, heated at $80{ }^{\circ} \mathrm{C}$; byield based on alkyl halide and determined by GC-MS.

The coupling reactions of other phenylboronic acid aryl compounds (1b and $\mathbf{1 c}$ ) with aryl halides were also studied using optimized reaction conditions. As shown in Table 3, substrates possessing electron-donating substituents worked well, returning the desired products in 91 and $89 \%$ yields (4a and $\mathbf{4 1}$ ). Substrates containing varying amounts of steric hindrance furnished the corresponding coupling products in 67 and $90 \%$ yields ( $\mathbf{4 b}$ and $\mathbf{4 c}$ ). Substrates with electron-withdrawing substituents gave $85 \%$ isolated yields (4s). However, neither 4-chlorophenylboronic acid nor 4-cyanophenyl boronic acid reacted with nitro-substituted substrates. Furthermore, the reactions between $1 \mathbf{c}$ and aryl halides were also investigated under identical conditions. 1c was similar to $\mathbf{1 b}$, highlighting the fact that most substituted alkyl bromides containing ether, amino, and nitrile groups are all well tolerated under these optimized reaction conditions, furnishing products in 95 and $86 \%$ yields (5b and 5d). Aryl halides also gave desired products in $62-92 \%$ yields without any interference from steric hindrance $\mathbf{( 5 c}$, $\mathbf{5 g}$, and $\mathbf{5 h}$ ), but 4-cyanophenyl boronic acid still failed to produce product in reasonable yields.

In addition, the catalytic performance of recycled Pd@PAAs-CD was studied, and the reusability of the catalyst system is attributed to the presence of $\beta-C D$, which protects the catalytic activity of Pd@PAAs-CD. The use of cyclodextrin and PAAs also made it easy to separate the product from the reaction mixture by simple extraction due to the heterogeneous nature of the catalyst system. Conversions demonstrating the recyclability of Pd@PAAs-CD in cross-coupling reactions are shown in Table 4, where Pd@PAAs-CD still retains high catalytic activity even after six successive reaction cycles with a conversion rate of $79 \%$.

\section{Conclusions}

In this paper, Pd NPs modified with PAAs-CD have been successfully synthesized via a facile method. It has been shown that Pd@PAAs-CD can act as an efficient and recyclable catalyst for Suzuki-Miyaura cross-coupling reactions in water with high yields and tolerate of a wide variety of functional groups. The source of this excellent catalytic activity is attributed to the special structure and properties of $\mathrm{CD}$ which is hydrophobic on its interior and hydrophilic on its exterior, which helps promote the catalytic activity of Pd@PAAs-CD through host-guest interactions. In addition, it was also demonstrated that Pd@PAAs-CD could be recycled and reused in cross-coupling reactions and showed only a minimal decrease in catalytic activity, even after six reaction cycles. The catalyst developed here can be applied to heterogeneous green chemical reactions and further studies of the applications of this hybrid material in selective catalysis are currently underway.

\section{Supplementary Information}

Supplementary information is available free of charge at http://jbcs.sbq.org.br as PDF file.

\section{Acknowledgments}

This work is supported by Shanghai Municipal Education Commission (Plateau Discipline Construction Program), National Science Foundation of China (No. 21601125 and 21174081), Natural Science Foundation of Shanghai (No. 16ZR1435700), Shuguang Scholar of Shanghai Municipal Education Commission (No. 16SG49), Shuguang Scholar Project and Start-up Funding of Shanghai Institute of Technology (YJ-201610).

\section{References}

1. Sherry, B. D.; Furstner, A.; J. Org. Chem. 2008, 41, 1500.

2. Ackermann, L.; Vicente, R.; Kapdi, A. R.; Angew. Chem., Int. Ed. 2009, 48, 9792.

3. Netherton, M. R.; Fu, G. C.; Adv. Synth. Catal. 2004, 346, 1525.

4. Tobisu, M.; Shimasaki, T.; Chatani, N.; Angew. Chem., Int. Ed. 2008, 47, 4866.

5. Yin, H.-Y.; Lin, X.-L.; Li, S.-W.; Shao, L.-X.; Org. Biomol. Chem. 2015, 13, 9012.

6. Zhu, Y.-Q.; Su, H.; Tang, J.-L.; Yang, A.-Q.; Comput. Theor. Chem. 2015, 1068, 47.

7. Chen, X.; Engle, K. M.; Wang, D.-H.; Yu, J.-Q.; Angew. Chem., Int. Ed. 2009, 48, 5094.

8. Lyons, T. W.; Sanford, M. S.; Chem. Rev. 2010, 110, 1147. 
9. Xu, H.-L.; Zhao, C.-L.; Qian, K.; Deng, W.; Gong, H.-G.; Chem. Sci. 2013, 4, 4022.

10. Olsson, V.-J.; Szabo, K.-J.; Angew. Chem., Int. Ed. 2007, 46, 6891.

11. Olsson, V. J.; Szabo, K. J.; Org. Lett. 2008, 10, 3129.

12. Scheuermann, G. M.; Rumi, L.; Steurer, P.; Bannwarth, W.; Mulhaupt, R.; J. Am. Chem. Soc. 2009, 131, 8262.

13. Crini, G.; Chem. Rev. 2014, 114, 10940.

14. Liu, M.-H.; Zhang, L.; Wang, T.-Y.; Chem. Rev. 2015, 115, 7304.

15. Herbois, R.; Noel, S.; Leger, B.; Bai, L.; Roucoux, A.; Monflier, E.; Ponchel, A.; Chem. Commun. 2012, 48, 3451.

16. Hapiot, F.; Menuel, S.; Ferreira, M.; Leger, B.; Bricout, H.; Tilloy, S.; Monflier, E.; ACS Sustainable Chem. Eng. 2017, 5, 3598.

17. Li, J.; Loh, X. J.; Adv. Drug Delivery Rev. 2008, 60, 1000.

18. Menand, M.; Sollogoub, M.; Boitrel, B.; le Gac, S.; Angew. Chem., Int. Ed. 2016, 55, 297.

19. Yu, G. C.; Jie, K. C.; Huang, F. H.; Chem. Rev. 2015, 115, 7240.

20. Ha, C. S.; Gardella, J. A.; Chem. Rev. 2005, 105, 4205.

21. Tan, S.; Ladewig, K.; Fu, Q.; Blencowe, A.; Qiao, G. G.; Macromol. Rapid Commun. 2014, 35, 1166.

22. Wu, L.; Zhang, Y.; Ji, Y.-G.; Curr. Org. Chem. 2013, 17, 1288.

23. Wang, J.; Wang, X. C.; Zhan, X. T.; J. Mater. Chem. A 2017, 5, 4308 .

24. Assaf, K.; Ural, M. S.; Pan, F.; Georgiev, T.; Simova, S.; Rissanen, K.; Gabel, D.; Nau, W. M.; Angew. Chem., Int. Ed. 2015, 54, 6852 .

25. Giacalone, F.; Campisciano, V.; Calabrese, C.; la Parola, V.; Syrgiannis, Z.; Prato, M.; Gruttadauria, M.; ACS Nano 2016, $10,4627$.

26. van Dongen, S. F. M.; de Hoog, H. P. M.; Peters, R. J. R. W.; Nallani, M.; Nolte, R. J. M.; van Hest, J. C. M.; Chem. Rev. 2009, 109, 6212.

27. Potier, J.; Menuel, S.; Monflier, E.; Hapiot, F.; ACS Catal. 2014, 4, 2342.
28. Li, H.-Y.; Meng, B.; Chai, S.-H.; Liu, H.-L.; Dai, S.; Chem. Sci. 2016, 7, 905.

29. Mori, K.; Yoshioka, N.; Kondo, Y.; Takeuchi, T.; Yamashita, H.; Green Chem. 2009, 11, 1337.

30. de Leener, G.; Evoung-Evoung, F.; Lascaux, A.; Mertens, J.; Porras-Gutierrez, A. G.; le Poul, N.; Lagrost, C.; Over, D.; Leroux, Y. R.; Reniers, F.; J. Am. Chem. Soc. 2016, 138, 12841.

31. Jia, H.; Schmitz, D.; Ott, A.; Pich, A.; Lu, Y.; J. Mater. Chem. A 2015, 3, 6187.

32. Kang, Y.; Zhou, L.-L.; Li, X.; Yuan, J.-Y.; J. Mater. Chem. 2011 , 21,3704 .

33. Putta, C.; Sharavath, V.; Sarkar, S.; Ghosh, S.; RSC Adv. 2015, $5,6652$.

34. Kaboudin, B.; Salemi, H.; Mostafalu, R.; Kazemi, F.; Yokomatsu, T.; J. Org. Chem. 2016, 818, 195.

35. Giacalone, F.; Campisciano, V.; Calabrese, C.; la Parola, V.; Syrgiannis, Z.; Prato, M.; Gruttadauria, M.; ACS Nano 2016, 10, 4627.

36. Herbois, R.; Noël, S.; Léger, B.; Tilloy, S.; Menuel, S.; Addad, A.; Martel, B.; Ponchela, A.; Monflier, E.; Green Chem. 2015, 17, 2444.

37. Zhang, W.; Yao, Z.-J.; Deng, W.; Chin. J. Org. Chem. 2018, 10, 2713.

38. Lai, W.-F.; Rogach, A. L.; Wong, W. T.; Chem. Soc. Rev. 2017, 46, 6379 .

39. Liu, J.; Liu, X.-X.; Yuan, Y.; Li, Q.-L.; Chang, B.-C.; Xu, L.-M.; Cai, C.; Qi, C.; Li, C.; Jiang, X.-L.; Wang, G.-B.; Wang, Z.; Wang, L.; ACS Appl. Mater. Interfaces 2018, 10, 26473.

40. Han, G.-D.; Wang, J.-T.; Ji, X. T.; Liu, L.; Zhao, H. Y.; Bioconjugate Chem. 2017, 28, 636.

41. Hu, Y.; Chai, M.-Y.; Yang, W.-T.; Xu, F.-J.; Bioconjugate Chem. 2013, 24, 1049.

Submitted: December 3, 2018

Published online: April 23, 2019 